Vol. 19, $n^{\circ} 1 \mid 2015$

Varia

\title{
Par-delà les frontières : nouvelles perspectives sur la recherche relative aux polices
}

Transcending Boundaries: New Approaches to the Study of Policing

Jonas Campion

\section{(2) OpenEdition \\ Journals}

Édition électronique

URL : http://journals.openedition.org/chs/1556

DOI : $10.4000 /$ chs. 1556

ISSN : 1663-4837

Éditeur

Librairie Droz

Édition imprimée

Date de publication : 1 juin 2015

Pagination : 113-118

ISBN : 978-2-600-01953-8

ISSN : $1422-0857$

\section{Référence électronique}

Jonas Campion, «Par-delà les frontières : nouvelles perspectives sur la recherche relative aux polices ", Crime, Histoire \& Sociétés / Crime, History \& Societies [En ligne], Vol. 19, n¹ | 2015, mis en ligne le 01 juin 2017, consulté le 24 septembre 2020. URL : http://journals.openedition.org/chs/1556 ; DOI : https://doi.org/10.4000/chs.1556 


\title{
Lecture croisée Review Essay
}

\author{
Par-delà les frontières: \\ nouvelles perspectives sur la recherche relative aux polices \\ Transcending Boundaries: \\ New Approaches to the Study of Policing
}

Vincent Denis, Catherine Denys (dir.), Polices d'Empires, XVIII ${ }^{e}-X I X^{e}$ siècles, Rennes, PUR, 2012, 194 pp., ISBN 978-2-7535-2074-5.

Catherine Denys (dir.), Circulations policières 1750-1914, Villeneuve d'Ascq, Presses Universitaires du Septentrion, «Temps, espace et société», 2012, 208 pp., ISBN 2-7574-0393-1.

Aurélien Lignereux, Servir Napoléon. Policiers et Gendarmes dans les départements annexés, 1795-1814, Seyssel, Champ Vallon, «Époques», 2012, 396 pp., ISBN 2-87673-624-5.

La recherche sur l'histoire des polices a, depuis quelques années, tendance à décloisonner les frontières géographiques de ses terrains d'études. Nous ne pouvons que nous en réjouir. De cette façon, se multiplient d'abord les approches croisées entre institutions de police; ensuite les recherches sur les multiples niveaux de coopérations policières qui s'organisent dès la fin du XIX ${ }^{\mathrm{e}}$ siècle, pour faire face à une criminalité nouvelle ou aux enjeux d'une police «politique $»^{1}$; enfin, les recherches interrogeant la réalité de la circulation et des transferts entre mondes policiers se développent, dépassant et nuançant le clivage traditionnel, apparu au XIX ${ }^{\mathrm{e}}$ siècle, entre modèles français et anglais de police. Ce renouvellement des perspectives illustre les processus d'influences réciproques, les adaptations qui apparaissent entre institutions de police de différentes cultures. Il a pour principal acquis de dorénavant prendre en compte le caractère évolutif et pluriel des influences en matière policière. En témoigne l'usage actuel du concept de «système», préféré à celui de «modèle » policier, dont la connotation est à la fois trop statique et trop réductrice, face à une pluralité des institutions et à la polysémie du concept de «police».

Dans ce questionnement sur les circulations des savoirs, pratiques et expertises ${ }^{2}$ policières, le renouveau de l'histoire coloniale a apporté des questionnements fertiles et des terrains d'observation propices. En effet, les nécessités de l'exercice de fonctions d'ordre et de justice dans les colonies ont entraîné une large dynamique de transferts et d'adaptations des institutions policières issues de la puissance

Nous renvoyons aux travaux précurseurs de Mathieu Deflem.

2 Qui, soulignons-le, ne touche pas uniquement l'histoire des polices mais, comme le démontre une rapide recherche sur Calenda, des domaines aussi variés que les savoirs, le jazz, les «collections», la musique et la littérature... Sur la discussion de ce concept, voir le numéro thématique de Critique internationale, 2013, t. 59, $\mathrm{n}^{\circ} 2$, consacré à la «Circulation des savoirs et champs transnationaux», et notamment la contribution d'Antoine Vauchez, «Le prisme circulatoire. Retour sur un leitmotiv académique», pp. 9-16. 
colonisatrice. Entre traditions et renouvellements, les contacts entre métropoles et colonies constituent des points cruciaux de la compréhension de l'exercice des fonctions de police: comment et avec qui maintenir l'ordre dans ces territoires de souveraineté récente? Les institutions policières sont-elles adaptées pour répondre aux spécificités coloniales (cohabitation de populations, protection des biens des colons, gestion de l'espace et des distances,...)? Dans quelle mesure les réalités coloniales influencent-elles ensuite la police telle qu'organisée en métropole?

Inscrites dans les développements récents de l'historiographie, ces modalités des circulations policières sont au cœur de différents projets éditoriaux, menés individuellement ou collectivement. Avec son Servir Napoléon. Policiers et gendarmes dans les départements annexés (1796-1814), Aurélien Lignereux s'intéresse au processus d'implantation et de structuration, au sein des départements annexés par la France révolutionnaire, du système français de police, structuré autour d'une gendarmerie et de polices municipales ${ }^{3}$. En publiant cette monographie, il clôture les recherches dont il égrenait les premiers résultats, depuis quelques années, de colloques en journées d'études. Dans la continuité du projet ANR Cirsap (Circulation des savoirs policiers en Europe $)^{4}$, Catherine Denys édite un éclairant Circulations policières 1750-1914, illustrant la typologie de la variété de modes de circulations de savoirs et pratiques entre forces de police, à la frontière entre époque moderne et période contemporaine. L'ouvrage souligne l'intérêt de réfléchir aux spécificités des chronologies policières, dépassant une périodisation sociopolitique trop traditionnelle. La même Catherine Denys dirige avec Vincent Denis, un Polices d'Empires, XVIII ${ }^{e}$-XIX ${ }^{e}$ siècles, éclairant ces problématiques dans une perspective où entrent en compte les rapports de domination induits par le processus de colonisation. Cette dernière contribution confirme les apports d'un dialogue métropole-colonie pour ce qui touche aux réalités policières.

Ces trois publications constituent autant d'aboutissements, réussis, de projets de longue haleine. Car ils mobilisent les spécialistes des sujets abordés; car ils s'inscrivent dans une perspective globale, et dépassent largement les cadres «traditionnels» des recherches menées en Europe francophone; car ils multiplient les éclairages géographiques (Asie, Afrique, Amériques) et envisagent un long $\mathrm{XIX}^{\mathrm{e}}$ siècle policier, transcendant les frontières entre Ancien Régime et période contemporaine, ces travaux représentent des apports majeurs au dynamisme du chantier de l'histoire des polices dans le monde.

Les perspectives de ces publications sont stimulantes à plus d'un titre, alors que les choix posés démontrent leur justesse. Leur lecture croisée illustre parfaitement les enjeux de la circulation des structures, pratiques et savoirs policiers entre pays, alors que se forment les États modernes. Pour en souligner les apports, il nous paraît utile de discuter de manière transversale certaines observations que l'on peut faire à partir de ces recherches, plutôt que de les présenter chacune de manière successive.

Si l'on désire, à partir de la variété de cas et de situations présentées, réfléchir à une typologie des circulations policières, le premier constat tient au caractère volontaire ou imposé des transferts. Indiscutablement, entre transfert raisonné

Sur ce sujet, nous renvoyons le lecteur au récent Jacques-Olivier Boudon (dir.), Police et gendarmerie dans l'Empire napoléonien, Paris, Éditions SPM, 2013, 242 p.

4 Sur ce projet actif entre 2006 et 2010, voir [http://irhis.recherche.univ-lille3.fr/ANR-CIRSAP.html]. Ses travaux ont débouché sur un nouveau programme intitulé «Systèmes policiers européens, $18^{\mathrm{e}}-19^{\mathrm{e}}$ siècles ». Voir [http ://syspoe.hypotheses.org/le-programme-syspoe]. 
et imposition policière, les moteurs des circulations sont variables, et largement nuancés. Cette question est évidemment au cœur des travaux d'Aurélien Lignereux qui étudie, à l'échelle de l'Europe, la construction de l'État et de ses services au sein des départements annexés par la France révolutionnaire puis par l'Empire. Elle est également constitutive des réalités coloniales. À l'opposé, les analyses présentes dans Circulations policières illustrent l'attrait de certains modes d'organisation policière, justifiant copie, implantation et appropriation volontaire. Les résultats de cette circulation policière doivent pourtant toujours être adaptés, tant est fondamental le rôle de la police dans la construction de l'État ou du pouvoir politique. D'une part, même lorsqu'un modèle policier est imposé sur un espace géographique, celuici subit une transformation plus ou moins assumée par rapport à l'idéal théorique initialement envisagé.

Comme l'a démontré à suffisance Aurélien Lignereux, la réalité policière est alors moins centralisatrice, unificatrice et uniforme que revendiquée dans les discours officiels. Réelle, la domination n'en est donc pas moins une adaptation (acclimatation?) aux diversités des situations locales. Si le discours officiel réfute de telles adaptations, pouvant être perçues comme des compromissions ou des faiblesses, la réalité est tout autre: souvent, il s'agit de tenir compte des possibilités locales du recrutement, des caractéristiques géographiques, ou de l'insuffisance des moyens financiers. Ce sont là autant de facteurs qui nécessitent de «régionaliser» un modèle théorique imposé. Cette dynamique d'adaptation est d'autant plus présente lorsque la circulation des systèmes policiers est volontaire. Même lorsqu'elle se revendique la plus proche possible du modèle originel, la circulation policière et les réformes qui en découlent sont en fait largement conditionnées par une réalité politique et sociale locale. L'inspiration est donc lue sous cette focale. Elle est alors instrumentalisée, comme un idéal à attendre ou au contraire, comme un repoussoir justifiant telle ou telle politique. Comme l'illustrent ces trois livres, toute circulation policière est donc avant tout une transformation. Adaptation de l'institution que l'on cherche à importer d'abord, adaptation des structures préexistantes d'autre part, qui sont loin de toujours disparaître purement et simplement avec l'apparition de nouvelles structures «modernes». Cette réalité mouvante illustre la place centrale qu'occupent les policiers et gendarmes dans le contrôle des populations, au service des autorités municipales, régionales ou nationales. À leur niveau, chacun de ces travaux pose la question de l'échec ou de la réussite des circulations, de la pérennité des réformes établies à leurs suites mais également, des causes structurelles ou circonstancielles qui les expliquent.

En questionnant les modes d'imposition d'un système policier, les auteurs abordent également la problématique de la violence inhérente à la police: celle exercée par les agents de l'ordre envers les populations, ainsi que les violences dont ils sont eux-mêmes victimes. Dans une situation de domination - qu'elle soit coloniale ou en vue d'une annexion - l'action policière est par définition répressive à l'égard des populations. Les formes de cette violence sont variées. Elle peut être basée sur la crainte, sur une dynamique de subsistance au détriment des populations, prendre la forme de colonnes armées, ou s'inscrire dans des doctrines de répression par les armes de tout mouvement séditieux ou de contestation. Les populations n'hésitent pas à résister à ces agents de l'État, ou à la domination qu'ils incarnent. Les formes de cette résistance sont multiples : à la passivité volontaire dans la transmission de renseignements utiles aux policiers, se rajoutent selon les cas des rebellions locales, ou des révoltes de plus grandes ampleurs. 
La gestion et la gradation de ces violences représentent des enjeux largement présents, notamment dans les ordres ou la formation des policiers. L'évolution des perceptions officielles à leur égard constitue alors un révélateur utile de la «normalisation», ou de la «pacification» d'un espace contrôlé. Il est intéressant d'envisager les influences entre métropoles et empires, dans la gestion de cette réalité ; mais aussi entre différentes régions d'un empire, ou entre systèmes policiers, pour nuancer plus en avant, la traditionnelle définition weberienne de la police comme titulaire légitime du monopole de la violence étatique.

Sous-jacente à la question du caractère imposé ou non des transferts, les contributions rassemblées par Catherine Denys dans Circulations policières soulignent la diversité des moyens d'appréhension et de circulation entre réalités policières. Voyages d'études, mémoires, «coopérations bilatérales», rapports personnels ou officiels, plaidoyers pro domo sont autant de moyens d'observation et d'assimilation des structures policières. Ils témoignent à la fois d'une généalogie du savoir (à analyser) et de la professionnalisation policière, mais aussi de l'apparition de réseaux transnationaux de relations, bâtis sur les hommes et les institutions, qu'il convient d'établir avec précision. Le phénomène ira en grandissant au cours du XX $\mathrm{X}^{\mathrm{e}}$ siècle, au fur et à mesure de la facilitation des communications et des voyages. En termes institutionnels et de compréhension des circulations, les rôles des policiers visiteurs et des policiers visités - dit autrement, des «modèles» et des «modelés » -, doivent être analysés pour comprendre les stratégies, les pratiques et les objectifs recherchés par les acteurs et services impliqués, dans des perspectives où se mêlent enjeux nationaux et transnationaux.

La qualité de ces trois ouvrages est de placer les acteurs - policiers, gendarmes, magistrats municipaux - au cœur des dynamiques de transferts et d'échanges policiers. Car étudier ces contacts ne peut faire l'économie des hommes qui les font vivre. Aux approches biographiques individuelles ${ }^{5}$, se rajoutent des approches collectives ou prosopographiques offrant des aperçus sociologiques sur les membres d'un corps (la gendarmerie de l'Empire, dans une perspective européenne), ou d'une police locale. Ces perspectives illustrent la diversité des parcours policiers, la diversité du recrutement (des «indigènes» pour la gendarmerie de l'Empire, des bons citoyens, des auxiliaires autochtones, mais aussi d'anciens forçats en Tasmanie/ Terre de Van Diemen). Apparaissent ainsi des facteurs structurants de l'exercice de fonctions de police: qualités professionnelles, qualités physiques et morales, mais également compétences linguistiques pour se faire comprendre des populations contrôlées. À ce profil idéal, transcendant les frontières, se confrontent les difficultés de la réalité, marquée par des enjeux financiers, de carrière, et les difficultés de la vie au sein des régions qu'il s'agit de contrôler (climat, volonté de se rapprocher de la famille, conditions de logement).

L'intérêt d'une approche "incarnée» tient également aux multiples niveaux d'échelles envisagés par les auteurs. Ils abordent à la fois des exemples issus de polices municipales, «nationales», régionales ou insulaires, mais aussi de corps de plus grande ampleur ou des institutions semi-privées dans certaines exploitations coloniales. Les profils policiers y sont multiples. Indéniablement, les circulations et échanges sont des réalités présentes au sein de l'ensemble des forces de police,

Piste en plein essor, dans l'historiographie policière. Voir les recherches menées au sein du Lectoraat PolitieGeschiedenis à Apeldoorn autour de Guus Meershoek. 
quelle que soit leur importance. Pour chaque situation, les auteurs illustrent le rapport à l'autorité, et plus largement le rapport à l'État qui sous-tend l'action des professionnels de l'ordre. Ils éclairent aussi les divers modes de régulations des populations contrôlées (qu'elles soient nationales ou indigènes), entre accommodation, police informelle, et les différentes modalités répressives.

Des villes de l'Ancien Régime aux colonies du XIX ${ }^{\mathrm{e}}$ siècle, en passant par le premier Empire français, les auteurs illustrent avec clarté et richesse, le caractère polysémique de la police et la diversité des tâches qui en découlent: à la fois juge officiel et agent de l'ordre sous l'Ancien Régime, mais aussi dans certaines situations coloniales où l'État peu présent est contraint d'accepter ce regroupement des fonctions. Contrôle des villes, contrôle des campagnes, lutte contre le brigandage, contre le refus de la conscription, police économique, police des exploitations sont autant de facettes de la quotidienneté des métiers de police qui sont abordées. Elles illustrent les réalités du maintien de l'ordre et de la tranquillité publique, au-delà des frontières.

Les résultats obtenus par ces auteurs invitent à élargir plus encore les perspectives chronologiques, tout en réfléchissant à la redéfinition des balises temporelles de l'histoire des polices. Comme le souligne Emmanuel Blanchard dans sa conclusion à Polices d'Empires, le cadre chronologique de l'étude des circulations policières mérite maintenant d'être élargi, notamment pour souligner les facteurs de permanences et de ruptures qui les traversent au gré des crises ou des transitions politiques $^{6}$. Pour les polices de l'Empire français, se pose la question du devenir des institutions après la chute de Napoléon. Comment les structures d'une part, les hommes de l'autre, vivent-ils la transition de l'après 1815? Plus largement, le vingtième siècle - notamment autour des guerres mondiales, de la décolonisation, et de la multiplication des interventions internationales sous égide de la SDN puis de l'ONU -, constitue une période où les dynamiques de circulation et de contacts policiers sont à analyser, en tenant également compte des transformations sociales, et de l'évolution des structures et missions de l'État.

Sans aucun doute, ces trois publications apportent des éléments nombreux et riches à la compréhension des circulations policières au XIX ${ }^{\mathrm{e}}$ siècle à l'échelle du monde. À ce titre, elles interrogent chacune les éléments constitutifs de l'apparition de systèmes modernes de police, en corollaire du renforcement des États-nations. Seul reproche possible, celui du déséquilibre parfois observé dans le traitement des différentes composantes des systèmes policiers dont il est question. Pour approfondir les enjeux des circulations en cours, le lecteur aimerait parfois mieux situer la pluralité des institutions policières en place à une époque donnée, entre polices judiciaires, civiles ou militaires. Malgré tout, le propos de ces trois études est clair et logiquement structuré pour envisager les différentes facettes de ces circulations et impositions de systèmes policiers dans une approche globale (organisation, adaptation, personnels, pratiques et missions). Signalons encore que tant la monographie d'Aurélien Lignereux, que les différentes contributions des ouvrages collectifs coordonnés par Catherine Denys et Vincent Denis offrent des bibliographies complètes et largement transnationales, outils dont l'intérêt est évident.

Questionnement présent dans le colloque «Empires: Social Control, Political Transition, (Post-) Colonial Legacies », organisé à Bruxelles en décembre 2013. 
Au final, il ne fait que peu de doutes que, par la richesse de leurs apports, chacun de ces livres a son importance dans l'historiographie policière. Ils mettent à disposition des chercheurs francophones (l'ensemble des contributions aux ouvrages collectives sont traduites), de pertinents points de comparaison, souvent méconnus, sur les différents continents pour sortir d'une historiographie trop souvent limitée par la barrière de la langue... En sens inverse, l'on peut espérer que ces recherches soient également traduites - en tout ou en partie ? - en anglais, pour les ouvrir à de plus larges publics et ainsi permettre une meilleure circulation des savoirs...

Jonas Campion Chargé de recherches FRS-FNRS Centre d'histoire du droit et de la justice UClouvain, Belgique PAI $7 / 22$ jonas.campion@uclouvain.be 